\title{
Determinants of CD4 cell count change and time-to default from HAART; a comparison of separate and joint models
}

\author{
Awoke Seyoum Tegegne ${ }^{1 *}$, Principal Ndlovu² and Temesgen Zewotir ${ }^{3}$
}

\begin{abstract}
Background: HIV has the most serious effects in Sub-Saharan African countries as compared to countries in other parts of the world. As part of these countries, Ethiopia has been affected significantly by the disease, and the burden of the disease has become worst in the Amhara Region, one of the eleven regions of the country. Being a defaulter or dropout of HIV patients from the treatment plays a significant role in treatment failure. The current research was conducted with the objective of comparing the performance of the joint and the separate modelling approaches in determining important factors that affect HIV patients' longitudinal CD4 cell count change and time to default from treatment.
\end{abstract}

Methods: Longitudinal data was obtained from the records of $792 \mathrm{HIV}$ adult patients at Felege-Hiwot Teaching and Specialized Hospital in Ethiopia. Two alternative approaches, namely separate and joint modeling data analyses, were conducted in the current study. Joint modeling was conducted for an analysis of the change of CD4 cell count and the time to default in the treatment. In the joint model, a generalized linear mixed effects model and Weibul survival sub-models were combined together for the repetitive measures of the CD4 cell count change and the number of follow-ups in which patients wait in the treatment. Finally, the two models were linked through their shared unobserved random effects using a shared parameter model.

Results: Both separate and joint modeling approach revealed a consistent result. However, the joint modeling approach was more parsimonious and fitted the given data well as compared to the separate one. Age, baseline CD4 cell count, marital status, sex, ownership of cell phone, adherence to HAART, disclosure of the disease and the number of follow-ups were important predictors for both the fluctuation of CD4 cell count and the time-to default from treatment. The inclusion of patient-specific variations in the analyses of the two outcomes improved the model significantly.

Conclusion: Certain groups of patients were identified in the current investigation. The groups already identified had high fluctuation in the number of CD4 cell count and defaulted from HAART without any convincing reasons. Such patients need high intervention to adhere to the prescribed medication.

Keywords: CD4 cell count change, GLMM, Heterogeneity, Homogeneity, Joint model, Time to default

\footnotetext{
* Correspondence: bisrategebrail@yahoo.com

${ }^{1}$ Department of statistics, Bahir Dar University, Bahir Dar, Ethiopia

Full list of author information is available at the end of the article
}

(c) The Author(s). 2018 Open Access This article is distributed under the terms of the Creative Commons Attribution 4.0 International License (http://creativecommons.org/licenses/by/4.0/), which permits unrestricted use, distribution, and reproduction in any medium, provided you give appropriate credit to the original author(s) and the source, provide a link to the Creative Commons license, and indicate if changes were made. The Creative Commons Public Domain Dedication waiver (http://creativecommons.org/publicdomain/zero/1.0/) applies to the data made available in this article, unless otherwise stated. 


\section{Background}

HIV is more prevalent in Sub-Saharan African countries like Ethiopia, and accounted for approximately $71 \%$ of world total of HIV infected people in 2013 [1, 2]. In Ethiopia the incidence rate was 1.5\% [3, 4]. As other regions in Ethiopia, the Amhara region, the study area, is highly affected by the disease [5].

Highly Active Antiretroviral Therapy (HAART) is a life time treatment therapy given to HIV infected individuals. The therapy is given as a combination of different medication drugs based on the mechanism of treating the viruses [6], and the usual measure of success or failure of the therapy is the patient's CD4 cell count. Other factors that can affect the CD4 cell count of patients on HAART are: age (aged patients have lower CD4 count responses to HAART [7-9]); sex (females have higher CD4 count responses to HAART [10]); and residential area (rural patients have lower CD4 cell count responses to HAART [11]). Some earlier scholars also showed that WHO HIV stages are a self-determining indicator for the variation or fluctuation of CD4 cell count change at the starting time of HAART [12]. Another study reported that there is a positive correlation between baseline CD4 cell count change and the CD4 cell count after the commencement of HAART $[13,14]$.

Earlier studies were conducted on the joint modeling of CD4 cell count change and time to default from the treatment considered that the CD4 cell count change as continuous variable [15] but the distribution of the CD4 count change, whatever it is, is discrete. This means the formal statistical results of [15] maybe invalid because of regarding discrete response CD4 cell count change as continuous. Furthermore, previous joint models were conventional linear mixed effect models with the assumption of constant within subject variance [16] a restrictive assumption which if violated by the data renders statistical analysis results invalid. The above mentioned shortcomings on what has been done on the joint modeling suggested considering the more flexible generalized linear mixed effect models in conjunction with parametric and semi parametric survival time models for the joint and separate modeling of CD4 cell count change and time to default from HAART to determine predictors of these patients' responses. As far as we are aware of the literature, no other such investigation has been conducted.

\section{Methods}

\section{Source of data}

The longitudinal data used in this study consists of records of $792 \mathrm{HIV}$ infected adult patients (at least 18 years old) enrolled at Felege Hiwot Teaching and Specialized Hospital, Amhara region, northwest Ethiopia. The hospital started the free HAART program in 2005 when there was limited ART service in public health institutions. However, the data consists of a record of patients with at least two follow-up visits as from September 2008 to August 2012 of the study period. Before starting HAART, patients were given health and HAART related education. Patients visited the Teaching and Specialized Hospital monthly for the first six months for HAART and thereafter quarterly for the remaining study period to get HAART medication for the subsequent months and for the review of the progression of their CD4 cell count change. An administrative permission was given by respective ethical committee of two universities namely Bahir Dar University Ethical approval committee (which belongs to Bahir Dar University), Ethiopia with Ref $\neq$ RCS/1412/2006 and School of Science Research Ethics Review Committee (which belongs to University of South Africa, South Africa, Ref\#2015-SSR-ERC_ 006, to use secondary data for current research. We can attach the written ethical statements up on request. The quality of data was controlled by the ART section of the hospital.

\section{Response variables in the data}

The response variable of interest, CD4 cell count change, was calculated from the laboratory determined CD4 cell counts of the patients at follow-up visits as:

$$
\Delta \mathrm{y}_{\mathrm{ij}}=\mathrm{y}_{\mathrm{ij}}-\mathrm{y}_{\mathrm{ij}-1}
$$

where $y_{i j}$ and $y_{i j-1}$ are the respective measured CD4 cell counts of patient $i$ at the $j^{\text {th }}$ and $(j-1)^{\text {th }}$ follow-up visits. The other response variable of interest was the time to default from HAART or equivalently the number of follow-up visits to default from HAART.

\section{Predictor variables in the data}

The time invariant predictors were: sex (Male, Female); residential area (Urban, Rural); level of education (No education, Primary, Secondary and Tertiary); marital status (Living with partner, Living without partner); level of income (Low, Middle and High); WHO stages of HIV (Stage1, Stage2, Stage3 and Stage4); ownership of cell phone (Yes, No), whether or not the patient disclosed the disease (Yes, No); age in years; and baseline CD4 cell count in cells $/ \mathrm{mm} 3$. Descriptive statistics of these variables are in the RESULTS section of this paper.

\section{The generalized linear mixed effects model}

Let $y_{i j}\left(i=1,2, \ldots, n ; j=1,2, \ldots, n_{i}\right)$ be the CD4 count change of patient $i$ at follow up visit time $j ; \boldsymbol{Y}_{\boldsymbol{i}}=\left(y_{i 1}, y_{i 2}, \ldots . y_{i n_{i}}\right)^{T}$ $; X_{i 1}^{T}$ be an $n_{i} \times p$ design matrix of fixed effects for patient $i$ that is associated with the $p$-dimensional vector $\boldsymbol{\beta}_{\mathbf{1}}$ of fixed effects; and $Z_{i 1}^{T}$ be an $n_{i} \times q$ design matrix of random effects for patient $i$ that is associated with the $q$ - 
dimensional vector $\boldsymbol{v}_{\boldsymbol{i}}$ of patient specific random effects. Then, if the conditional distribution of $\boldsymbol{Y}_{\boldsymbol{i}}$ given $\boldsymbol{\nu}_{\boldsymbol{i}}$ is from the exponential family, the generalized linear mixed effects model for $\boldsymbol{Y}_{\boldsymbol{i}}$ can be written as:

$$
g\left(\boldsymbol{Y}_{\boldsymbol{i}}\right)=\boldsymbol{X}_{i 1}{ }^{T} \boldsymbol{\beta}_{1}+\boldsymbol{Z}_{i 1}^{T} \boldsymbol{\nu}_{\boldsymbol{i}}+\boldsymbol{\varepsilon}_{i}
$$

where $g($.$) is the link function that is completely specified$ by specifying the conditional of $\boldsymbol{Y}_{\boldsymbol{i}}$ in the exponential family. For example, $g()=.\log ($.$) if the distribution is Poisson$ as is possibly the case with the CD4 cell count change. Usually, $\boldsymbol{v}_{\boldsymbol{i}}$ is assumed to be multivariate normally distributed with mean vector zero and covariance matrix, $\boldsymbol{v}_{\boldsymbol{i}}$, and $\boldsymbol{\varepsilon}_{\boldsymbol{i}}$ assumed to be multivariate normally distributed with mean vector zero and covariance matrix $\boldsymbol{I} \sigma_{\varepsilon}^{2}$. The term $\boldsymbol{Z}_{i 1}^{T} \boldsymbol{v}_{\boldsymbol{i}}$ in model (2) accounts for patient level variation in CD4 cell count change. Model (2) was fitted to the data using Proc glimmix in SAS Version 9.2.

\section{Survival time models}

Let $t_{j}(j=1,2, \ldots, n)$ be the time to default from HAART for patient $i$; and $\boldsymbol{X}_{i 2}^{T}=\left(x_{12}, x_{22}, \ldots x_{k 2}\right)$ be a $p$-dimensional fixed effects vector of covariates for patient $i$ that is associated with the $p$-dimensional vector $\boldsymbol{\beta}_{2}$ of fixed effects. Then both the parametric and the semi parametric models for $t_{j}$ have hazard functions at the time $t$ of the form:

$$
h_{i}(t)=h_{0}(t) \exp \left[\boldsymbol{X}_{i 2}{ }^{T} \boldsymbol{\beta}_{2}\right]
$$

with the difference that parametric models specify the baseline hazard function $h_{0}(t)$ (or equivalently the distribution of $t_{j}$ ). The usual specified distributions include the Weibull, the Exponential, the Log logistic and the Log normal. For example, when the distribution is Weibull model (2) becomes the parametric model:

$$
h_{i}(t)=\varnothing \rho t^{\rho-1} \exp \left[\boldsymbol{X}_{i 2}{ }^{T} \boldsymbol{\beta}_{2}\right]
$$

where $\varnothing$ is the dispersion parameter and $\rho$ is the shape parameter of the distribution. The semi-parametric model (unspecified to $h_{0}(t)$ ) is the widely used Cox Proportional Hazards $(\mathrm{PH})$ model [17]. The parametric and semi-parametric models were fitted to the data using Proc glimmix in SAS Version 9.2.

In this study, the direct formulation of joint modeling of both CD4 cell count change and time to default from HAART with the introduction of Bayesian perspective within Markov Chain Monte Carlo (MCMC) structures [18] was adopted. The generalized linear mixed model for $\mathrm{CD} 4$ cell count change becomes:

$$
g\left(\boldsymbol{Y}_{\boldsymbol{i}}\right)=\boldsymbol{X}_{i 1}{ }^{T} \boldsymbol{\beta}_{1}+\boldsymbol{Z}_{i 1}^{T} \boldsymbol{\nu}_{\boldsymbol{i}}+\boldsymbol{\varepsilon}_{i}
$$

as in model (2) but with the assumption that $\boldsymbol{\varepsilon}_{i} \sim N\left(\mathbf{0}, b_{i} I\right)$ and $\log \left(b_{i}\right) \sim N\left(0, \sigma_{b}^{2}\right)$ [19]. Here, $b_{i}$ denotes for the actual variability for specific- patients which follows a log-normal distribution with mean 0 and variance $\sigma_{b}^{2}$ [20].

The corresponding survival time model is expressed as:

$$
h_{i}(t)=h_{0}(t) \exp \left[\boldsymbol{X}_{i 2}{ }^{T} \boldsymbol{\beta}_{2}\right]+W_{i 2}
$$

where $W_{\boldsymbol{i} 2}=\boldsymbol{v}_{\boldsymbol{i}}^{\boldsymbol{T}} \boldsymbol{\tau}+\tau_{q+1} \log b_{i}+v_{q+1}$ (with $\boldsymbol{v}_{\boldsymbol{i}}$ and $b_{i}$ as defined in model (5), $\left(\boldsymbol{\tau}^{T}, \tau_{q+1}\right)$ a vector of parameters, and $v_{q+1} \sim N\left(0, \sigma_{v}^{2}\right)$, a frailty effect is an added heterogeneity term to model (3) in order to account for communal and patient specific random effects. In this context model (4) becomes:

$$
h_{i}(t)=\varnothing \rho t^{\rho-1} \exp \left[\boldsymbol{X}_{i 2}^{T} \boldsymbol{\beta}_{2}\right]+W_{i 2}
$$

Thus, the models for CD4 cell count change and time to default from HAART are correlated through the random effect models:

$$
W_{i 1}=Z_{i 1}^{T} v_{i}
$$

and

$$
W_{i 2}=\boldsymbol{v}_{\boldsymbol{i}}^{T} \boldsymbol{\tau}+\tau_{q+1} \log b_{i}+v_{i q+1}
$$

In this study, $\boldsymbol{v}_{\mathrm{i}}=\left(v_{\mathrm{i} 0}\right) \mathrm{T}$ is the random of patient i's effect and $v_{02}$ is the random fraility term.

\section{Joint model selection}

The specific nature of random effect models (7) and (8) is selected using the Deviance Information Criterion (DIC) $[15,21]$ which is a hierarchical form of common Akaike Information Criteria (AIC) [22]. As with the AIC, a model with the smallest DIC is preferred.

\section{Results}

The data was analyzed using SAS Version 9.2. Table 1 displays the summary statistics of the predictor variables in the data. Among the sample of 792 patients: 40.9\% were rural residents; $50.6 \%$ were females; $44.8 \%$ were living with their partner; $72.6 \%$ of them disclosed their disease; $50.5 \%$ were owners of cell phone; and $68.2 \%$ of the patients had good adherence to HAART.

A decision had to be made on whether or not the conditional distribution of the CD4 cell count change was standard Poisson, quasi-Poisson or negative Binomial.

Figure 1 displays the graph of the average CD4 cell count change versus the corresponding standard deviation at each follow-up visit time. The graph shows that at each visiting time, the variance was greater than the mean which suggested that the distribution of the CD4 cell count change was over dispersed, and hence could be either be negative Binomial or quasi-Poisson. The quasi-Poisson model was preferred, because the average CD4 cell count change was greater than the cut-off point [14], and because the comparison of the negative Binomial and the quasi-Poisson 
Table 1 Descriptive statistics of potential predictor variables of CD4 cell count change and time to default from HAART in the data sample size 792

\begin{tabular}{|c|c|c|c|}
\hline \multicolumn{2}{|l|}{ Variable } & Average & No (\%) \\
\hline \multicolumn{2}{|l|}{ Weight (kg) } & $62(58,70)$ & - \\
\hline \multicolumn{2}{|l|}{ Baseline CD4 count cells/ $\mathrm{mm}^{3}$} & $150(113,198)$ & - \\
\hline \multicolumn{2}{|l|}{ Age (years) } & $36(28,48)$ & - \\
\hline \multicolumn{2}{|c|}{ First month / initial CD4 cell count change $/ \mathrm{mm}^{3}$} & $15.9(12-26)$ & - \\
\hline \multirow[t]{2}{*}{ Sex } & Male & & $391(49.4)$ \\
\hline & Female & & $401(50.6)$ \\
\hline \multirow[t]{4}{*}{ Educational status } & no education & & $160(20.2)$ \\
\hline & Primary & & $205(25.9)$ \\
\hline & Secondary & & $273(34.5)$ \\
\hline & Tertiary & & $154(19.4)$ \\
\hline \multirow[t]{2}{*}{ Residential area } & Urban & & $468(59.1)$ \\
\hline & Rural & & $324(40.9)$ \\
\hline \multirow[t]{2}{*}{ Marital status } & Living with partner & & $355(44.8)$ \\
\hline & Living without Partner & & $437(55.2)$ \\
\hline \multirow[t]{3}{*}{ Level of Income } & Low income (< 500 ETB per month) & & $355(44.8)$ \\
\hline & Middle income (5001-999 ETB per month) & & $346(43.7)$ \\
\hline & High income ( $\geq 1000$ ETB per month) & & $91(11.5)$ \\
\hline \multirow[t]{4}{*}{ WHO HIV Stage } & Stage I & & $101(12.8)$ \\
\hline & Stage II & & $258(32.6)$ \\
\hline & Stage III & & $199(25.1)$ \\
\hline & Stage IV & & $234(29.5)$ \\
\hline \multirow[t]{2}{*}{ Disclosure } & Yes & & $575(72.6)$ \\
\hline & No & & $217(27.4)$ \\
\hline \multirow[t]{2}{*}{ Cell ownership } & Yes & & $400(50.5)$ \\
\hline & No & & $392(49.5)$ \\
\hline \multirow[t]{3}{*}{ First month HAART adherence } & Good & & $540(68.2)$ \\
\hline & Fair & & $160(20.2)$ \\
\hline & Poor & & 92 (11.6) \\
\hline
\end{tabular}

model using the information criteria statistics (the smaller is the better) in Table 2 favored the quasi-Poisson model.

To fit the survival model, the parametric (Weibul models) and semi-parametric (Cox proportional hazard Model) models were take in to consideration and the two models were compared using AIC. As usual, the goodness of fit of the Weibul and Cox Proportional models were compared using a test statistics such as the Akakai information criteria (AIC) and Bayesian information criteria (BIC) and Pearson Chi-square divided by degree of freedom, assuming the smaller value as the better one. The comparison between survival models (Weibul and Cox proportional models) is shown in Table 3.

Table 3 indicates that Weibul has smaller Pearson chisquare/d.f, AIC and BIC. Hence Weibul was in favor of Cox proportional hazards model and it fitted the data well as compared to proportional hazards model.
Models (2) and (5) were fitted to the data using Proc glimmix in SAS Version 9.2. The two models are shown in Table 4, and are not very different from each. However, model (5) fitted the data better because model (5) has smaller posterior estimates for many of the predictors.

Estimation of parameter in both the full Weibull and exponential models were similar to each other, but the estimated Weibull shape parameter, $\rho$ was 0.763 with $95 \% \mathrm{CI}$ $(0.484,0.990)$ which is less than 1 and indicates that default rate decreased as the number of follow-ups increased.

In view of the fact that results and conclusions of this study will be most valid and reliable if the missing observations (due to patient dropouts/defaulters) are missing completely at random (MCAR), the data was analyzed as described in Table 4 to assess the data missing mechanism. A logistic regression model with $0=$ missing and $1=$ not missing responses was fitted to the data to obtain 


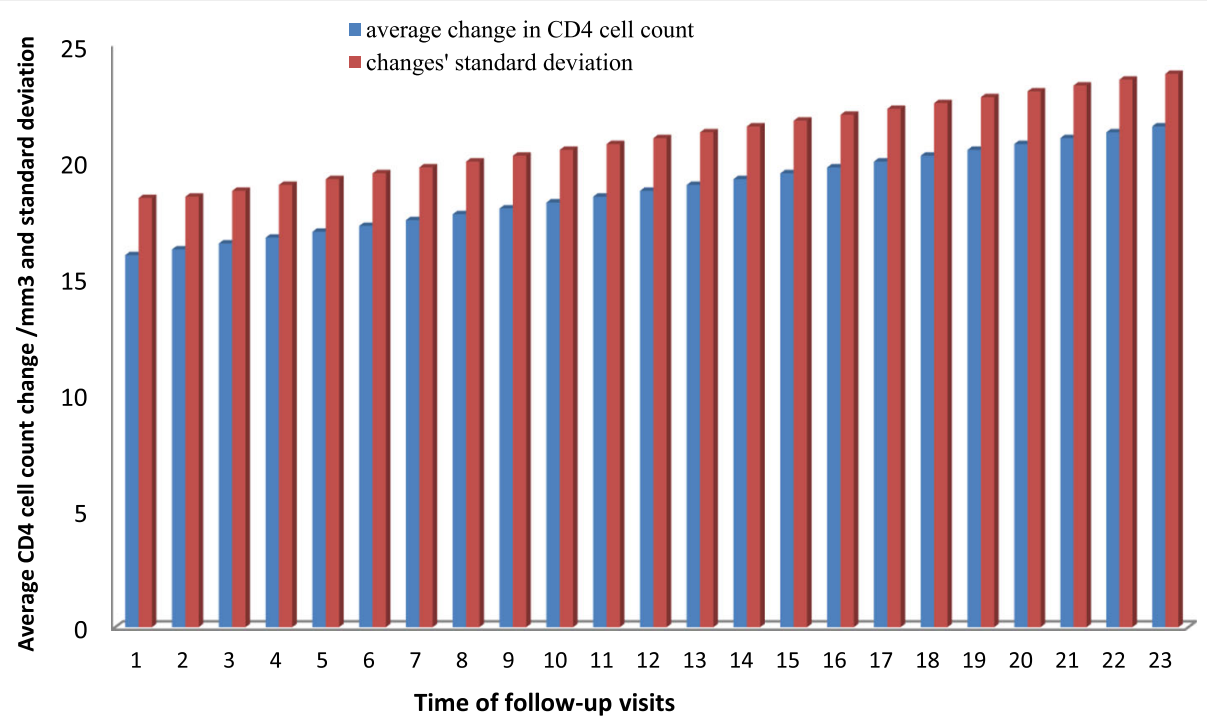

Fig. 1 The average CD4 cell count change versus the corresponding standard deviation at each follow-up visits

Table 5. All the effects are insignificant which suggests that MCAR was the data missing mechanism.

\section{Joint model results}

First the separate data analyses were conducted and then two joint models with different latent process were conducted for the fluctuation of repetitive CD4 cell count. The first were joint models with homogeneous variance assumptions using (1) and the other was joint models with heterogeneous variance of CD4 count change for each individual (5). Hence, the longitudinal sub model was described by both the common/conventional generalized linear mixed effects model and by the generalized linear mixed effects model including patient-explicit variances. Alternatively, the time to default from the HAART sub-model was fitted using a full Weibul distribution and the two sub-models were related applying communal covariates.

Based on the baseline variables, the longitudinal sub models were constructed using the usual generalized linear mixed effect model with the assumption of homogeneous patient specific CD4 cell count change and log likelihood function was reduced as it was done in the joint models with inclusion of different random effects and different forms of latent processes $W_{1}(\mathrm{t})$ and $W_{2}$

Table 2 Comparison of quasi-Poisson and negative Binomial models using information criteria

\begin{tabular}{llllllll}
\hline Criterion & \multicolumn{3}{l}{ quasi-Poisson } & & \multicolumn{3}{c}{ negative-Binomial } \\
\cline { 2 - 4 } & Value & d.f & Value/d.f & & Value & d.f & Value/d.f \\
\hline Pearson Chi-square & 1309 & 773 & 1.693 & & 1530 & 773 & 1.98 \\
Log likelihood & -2159 & & & -2658 & & \\
AIC & 4355 & & & 5354 & & \\
BIC & 4444 & & & 5443 & & \\
\hline
\end{tabular}

(t). In constructing joint models, the simple joint model (model, (2)) with no random effect in the two sub model was conducted. Next, joint models with random intercept $v_{0}$ and a frailty term $v_{3}$ were constructed successively. The inclusion of a frailty term, $v_{3}$ in the time to default sub-model, leads for improvement of the model. Hence, DIC decreased as frailty terms included in the time-to default sub model $W_{2}(\mathrm{t})$.

The correlation between $W_{1}(\mathrm{t})$ and $W_{2}(\mathrm{t})$ was introduced using communal random intercept, $v_{0}$ and this indicates that the DIC further decreased. In addition to the random intercept, the random slope was also included in the longitudinal data analysis. The inclusion of random slope also reduced the DIC. The result of this subsequent reduction of DIC is indicated in Table 6.

Table 6 indicates that the inclusion of random intercept as well as random intercept and slopes substantially reduced the DIC.

On the other hand, the generalized linear mixed effect model with the inclusion of subject-explicit CD4 cell count change variability in longitudinal data analysis was conducted. These joint models relate the variability existed in CD4 cell count changes

Table 3 Comparison of Weibul and Cox regression models using information criteria

\begin{tabular}{|c|c|c|c|c|c|c|}
\hline \multirow[t]{2}{*}{ Criterion } & \multicolumn{3}{|c|}{ Weibul model } & \multicolumn{3}{|c|}{ Cox regression model } \\
\hline & Value & d.f & Value/d.f & Value & d.f & Value/d.f \\
\hline Pearson Chi-square & 1409 & 773 & 1.83 & 1630 & 773 & 2.11 \\
\hline Log likelihood & -2059 & & & -2658 & & \\
\hline $\mathrm{AIC}$ & 4255 & & & 5454 & & \\
\hline $\mathrm{BIC}$ & 4544 & & & 5543 & & \\
\hline
\end{tabular}


Table 4 Posterior means and correspondence $p$-values for parameter estimation with inclusion and exclusion of patient specific variance

\begin{tabular}{|c|c|c|c|c|}
\hline \multirow[t]{2}{*}{ Effect } & \multicolumn{2}{|c|}{ Model (2): With homogeneity of variance for patients } & \multicolumn{2}{|c|}{ Model (5): With patient specific variance/heterogeneity } \\
\hline & Posterior estimate & $p$-value & Posterior estimate & $p$-value \\
\hline Intercept & 4.350 & 0.004 & 4.130 & 0.002 \\
\hline Age & -0.006 & 0.003 & -0.036 & 0.005 \\
\hline Weight & 0.001 & 0.082 & 0.001 & 0.082 \\
\hline Baseline CD4 & 0.005 & 0.008 & 0.002 & 0.008 \\
\hline \multicolumn{5}{|l|}{ Area (Reference = Urban) } \\
\hline Rural & -0.002 & 0.064 & -0.003 & 0.003 \\
\hline \multicolumn{5}{|c|}{ Marital Status (Reference $=$ without partner) } \\
\hline With partner & 0.007 & 0.003 & 0.005 & 0.003 \\
\hline \multicolumn{5}{|l|}{ Sex (Reference $=$ male) } \\
\hline Female & 0.034 & 0.004 & 0.023 & 0.003 \\
\hline \multicolumn{5}{|c|}{ Level of education (Reference $=$ Tertiary) } \\
\hline No educationl & 0.001 & 0.076 & 0.001 & 0.076 \\
\hline Primary education & 0.017 & 0.044 & 0.017 & 0.034 \\
\hline Secondary education & 0.020 & 0.028 & 0.010 & 0.038 \\
\hline \multicolumn{5}{|c|}{ Level of Income (Reference = High income) } \\
\hline Low income & -0.010 & 0.074 & -0.020 & 0.064 \\
\hline Middle income & -0.006 & 0.062 & -0.016 & 0.052 \\
\hline \multicolumn{5}{|c|}{ Owner of Cell phone (Reference $=$ With phone) } \\
\hline Without cell phone & -0.001 & 0.004 & -0.001 & 0.004 \\
\hline \multicolumn{5}{|c|}{ Level of adherence (Reference = Good adherence) } \\
\hline Poor adhere & -0.523 & 0.004 & -0.483 & 0.002 \\
\hline Fair adhere & -0.452 & 0.001 & -0.552 & 0.002 \\
\hline \multicolumn{5}{|c|}{ Level of disclosure the disease (Reference = yes) } \\
\hline No & -0.00195 & 0.0031 & -0.1020 & 0.0031 \\
\hline \multicolumn{5}{|c|}{ WHO stages (Reference $=$ Stage IV) } \\
\hline Stage 1 & 0.1511 & 0.0021 & 0.2511 & 0.0021 \\
\hline Stage II & 0.1567 & 0.0321 & 0.1567 & 0.0321 \\
\hline Stage III & 0.1381 & 0.2311 & 0.4381 & 0.0311 \\
\hline Time & 0.0210 & 0.0013 & 0.1210 & 0.0213 \\
\hline $\operatorname{Var}\left(v_{i 0}\right)$ & 0.8820 & & 0.8620 & \\
\hline $\operatorname{Var}\left(v_{i 1}\right)$ & 0.0210 & & 0.0110 & \\
\hline $\operatorname{Cov}\left(v_{i 0}, v_{i 1}\right)$ & -0.0542 & & -0.0642 & \\
\hline $\operatorname{Corr}\left(v_{\mathrm{i} 0}, v_{\mathrm{i} 1}\right)$ & -0.8214 & & -0.7314 & \\
\hline DIC & 234,763 & & 212,393 & \\
\hline
\end{tabular}

$v_{i 0}$ is the random of patient $i$ effect and $v_{i 1}$ is the random fraility term

to the follow-up visits defaulting from HAART. This relation shows that fluctuation(ups and downs) of the longitudinal outcome (CD4 cell count change) can be quantified as hazard ratio [23]. Hence, the inclusion of patient/subject specific CD4 cell count fluctuation in joint model improves the model to fit the data well. The selected model indicates that patient's survival was related to the increase of rate of
CD4 cell count and decrease of its fluctuation/variability of CD4 cell count which further indicates that the increase of CD4 count change for a particular patient leads to better health status and such patient has less likely in defaulting from treatment. While, patients with high variability of CD4 cell count change leads to poor health conditions and has more likelihood in defaulting from HAART. 
Table 5 Posterior effects of the predictor variables from modeling CD4 cell count change with models (2) (no patient specific variance) and (5) (with patient specific variance)

\begin{tabular}{|c|c|c|c|}
\hline Parameters & Estimate & Standard error & $P$-values \\
\hline Intercept & 1.808 & 0.910 & 0.470 \\
\hline Age & -0.658 & 0.145 & 0.831 \\
\hline Follow up times & 0.047 & 0.052 & 0.058 \\
\hline \multicolumn{4}{|c|}{ Previous CD4 cell count $\left(r e f=y_{j} \leq y_{j-1}\right)$ or $l_{i}=0$} \\
\hline$l_{i}=1 \quad\left(y_{j}>y_{j-1}\right)$ & 0.072 & 0.320 & 0.650 \\
\hline \multicolumn{4}{|l|}{ Gender (ref = female) } \\
\hline Male & 0.003 & 0.084 & 0.976 \\
\hline \multicolumn{4}{|c|}{ Residence area (ref = Urban) } \\
\hline Rural & -0.380 & 0.083 & 0.648 \\
\hline \multicolumn{4}{|c|}{ Marital status(ref = living without partner) } \\
\hline Living with partner & 0.109 & 0.086 & 0.208 \\
\hline
\end{tabular}

\section{Separate and joint model comparisons}

Considering the final selected joint model, the results obtained from this model was compared to the separated models (without latent association introduced by $\mathrm{W}_{2}$ ). The models with subject specific CD4 cell count change variability had smaller DIC as compared to models without subject specific CD4 cell count variability. To compare the joint and separate models, first subject specific CD4 cell count change incorporated the variability to both models were considered. The comparison of the two approaches was indicated in Table 7. During comparison, only significant predictors at separate models were considered.

Table 7 indicates that, the posterior estimates of the correlated parameters at joint model analysis were considerably different from zero and this is an indication of the correlation between the two sub models. The estimate of the shape parameter in the CD4 cell count fluctuation is negative $\left(\tau_{1}=-2.324\right)$ which indicates that the increase in the fluctuation of the CD4 cell count change was negatively associated with the number of follow-up visits in HAART. The positive value of association for parameters in the CD4 cell count change variability indicates that there is a direct proportionality between the CD4 cell count change fluctuation and the hazard of defaulting from HAART. Hence, as the CD4 cell count change variability increased, the hazard of defaulting also increased.
The convergence of the final joint models was checked with time series of iterations. There was a higher degree of randomness between successive iterations and this indicates that the value converged to a particular target density. The estimated hazard ratio and 95\% credible intervals for the joint survival and CD4 cell count change data were indicated in Table 8.

As presented in Table 8, some covariates such as age, baseline CD4 cell count, marital status, cell phone ownership, adherence level, disclosure's level of disease and the number of follow-ups of patients had significant effect on both the repetitive measures of the CD4 cell count fluctuation and the number of followed-up visits required to default from HAART. Hence, as age of a patient increased, the CD4 cell count change as well as its waiting time in the HAART decreased. However, whenever a patient started his/her HAART with a relatively high baseline CD4 cell count, the number of CD4 cell count and waiting time in the HAART also increased. Patients without the ownership of cell phone had decreased by $0.01\left(e^{-0.006}\right)$ his/her improvement of CD4 cell count change as compared to patients who owned cell phone. Fair adherent patients had $33 \%$ less probability to have improvement in their CD4 cell count as compared to good adherent patients. Similarly, poor adherent patients had $41 \%$ less likelihood to have improved their CD4 cell count as compared to good adherent patients. Such poor adherent patients had a short waiting time in the HAART. As the visiting times/ the number of follow-ups visits of a patient increased by one unit, the improvement of his/her CD4 cell count increased by $2.1 \%$, keeping the other variables constant. Female patients had $3.4 \%$ improvement in their CD4 cell count change as compared to males.

\section{Discussions}

The study was conducted using a Bayesian approach to jointly model the CD4 cell count change and the time to default from HAART. The results of the current study indicate that the joint model was in favor of separate models for determining the predictors of CD4 cell count change and time to default. This outcome is complemented by the results of previous research [15] . Another study stated that joint models showed a significant difference between treatment groups that was not identified by the separate model data analyses [18]. Since the correlated parameters in the communal random effect

Table 6 Model selection for joint data analysis using generalized linear mixed effect for longitudinal data and Weibul Survival models for survival data

\begin{tabular}{llll}
\hline Random Effects & $W_{1}(\mathrm{t})$ & $\mathrm{W}_{2}(\mathrm{t})$ & $\mathrm{DIC}$ \\
\hline Only fixed effect & 0 & 0 & 3547 \\
Fixed effect +random intercepts only & $V_{0}$ & 0 & 3506 \\
Fixed effect +Random intercept+ random intercepts and slopes & $V_{0}+v_{1}(\mathrm{t})$ & $\tau_{0} v_{0}+\tau_{1} v_{1}+v_{3}$ \\
\hline
\end{tabular}


Table 7 A separate and joint model comparison for longitudinal CD4 cell count change and time to default from HAART

\begin{tabular}{|c|c|c|c|c|}
\hline \multirow[t]{2}{*}{ parameter } & \multicolumn{2}{|l|}{ Separate models } & \multicolumn{2}{|l|}{ Joint models } \\
\hline & Posterior mean & $p$-value & Posterior mean & $p$-value \\
\hline \multicolumn{5}{|c|}{ Longitudinal sub models } \\
\hline Intercept & 4.350 & 0.004 & 4.130 & 0.002 \\
\hline Age & -0.006 & 0.003 & -0.036 & 0.005 \\
\hline Weight & 0.001 & 0.082 & 0.001 & 0.082 \\
\hline Baseline CD4 & 0.004 & 0.008 & 0.002 & 0.008 \\
\hline \multicolumn{5}{|c|}{ Marital Status (Reference $=$ without partner) } \\
\hline With partner & 0.007 & 0.003 & 0.005 & 0.003 \\
\hline \multicolumn{5}{|l|}{ Sex (Reference $=$ male) } \\
\hline Female & 0.034 & 0.004 & 0.023 & 0.003 \\
\hline \multicolumn{5}{|c|}{ Ownership of cell phone (Reference $=$ With phone) } \\
\hline Without cell phone & -0.001 & 0.004 & -0.001 & 0.004 \\
\hline \multicolumn{5}{|c|}{ Level of adherence (Reference = Good adherence) } \\
\hline Poor adherence & -0.523 & 0.004 & -0.483 & 0.002 \\
\hline Fair adherence & -0.452 & 0.001 & -0.552 & 0.002 \\
\hline \multicolumn{5}{|c|}{ Level of disclosure of the disease (Reference $=$ yes) } \\
\hline No & -0.002 & 0.003 & -0.102 & 0.003 \\
\hline \multicolumn{5}{|c|}{ WHO stages (Reference $=$ Stage IV) } \\
\hline Stage 1 & 0.151 & 0.002 & 0.251 & 0.002 \\
\hline Stage II & 0.1567 & 0.032 & 0.157 & 0.032 \\
\hline Stage III & 0.1381 & 0.231 & 0.438 & 0.031 \\
\hline Time & 0.021 & 0.001 & 0.121 & 0.021 \\
\hline $\operatorname{Var}\left(V_{0 i}\right)$ & 0.882 & 0.032 & 0.862 & 0.023 \\
\hline $\operatorname{Var}\left(V_{1 i}\right)$ & 0.021 & 0.043 & 0.011 & 0.005 \\
\hline $\operatorname{Cov}\left(V_{0 i}, V_{1 i}\right)$ & -0.054 & 0.032 & -0.064 & 0.006 \\
\hline $\operatorname{Corr}\left(V_{0 i}, V_{1 i}\right)$ & -0.821 & 0.021 & -0.731 & 0.001 \\
\hline \multicolumn{5}{|l|}{ Survival sub models } \\
\hline Intercept & 1.202 & 0.003 & 1.402 & 0.004 \\
\hline Age & -0.006 & 0.003 & -0.036 & 0.005 \\
\hline Baseline CD4 & 0.004 & 0.008 & 0.002 & 0.008 \\
\hline \multicolumn{5}{|c|}{ Marital Status (Reference = without partner) } \\
\hline With partner & 0.007 & 0.003 & 0.005 & 0.003 \\
\hline \multicolumn{5}{|l|}{ Sex (Reference = male) } \\
\hline Female & 0.034 & 0.004 & 0.023 & 0.003 \\
\hline Without cell phone & -0.001 & 0.004 & -0.001 & 0.004 \\
\hline \multicolumn{5}{|c|}{ Level of adherence (Reference = Good adherence) } \\
\hline Poor adherence & -0.523 & 0.004 & -0.483 & 0.002 \\
\hline Fair adherence & -0.452 & 0.001 & -0.552 & 0.002 \\
\hline \multicolumn{5}{|c|}{ Level of disclosure the disease (Reference = yes) } \\
\hline No & -0.002 & 0.003 & -0.102 & 0.003 \\
\hline \multicolumn{5}{|c|}{ WHO stages (Reference $=$ Stage IV) } \\
\hline Stage I & 0.151 & 0.002 & 0.251 & 0.002 \\
\hline Stage II & 0.157 & 0.032 & 0.157 & 0.032 \\
\hline Stage III & 0.138 & 0.231 & 0.438 & 0.031 \\
\hline
\end{tabular}


Table 7 A separate and joint model comparison for longitudinal CD4 cell count change and time to default from HAART (Continued)

\begin{tabular}{lllll}
\hline parameter & Separate models & Joint models \\
\cline { 2 - 3 } & Posterior mean & $p$-value & Posterior mean & 0.121 \\
\hline Time(visiting time) & 0.021 & 0.001 & -2.324 & 0.021 \\
$\tau_{1}$ & & & 0.051 & 0.005 \\
$\tau_{3}$ & & & 0.864 & 0.003 \\
$\rho$ & 0.654 & 0.004 & 0.003 \\
\hline
\end{tabular}

models measure the link between the two sub models, the associated information or common predictors of the repetitive outcomes and the time to default from HAART can be identified easily. The joint model had a smaller posterior mean as compared to the separate models and this indicates that the joint model fits the data well as compared to the separate models. The inclusion of subject specific variability in longitudinal CD4 cell count change improved the model significantly. Elder patients had higher CD4 cell count change fluctuation as compared to youngsters and this indicates that elders had low CD4 cell count change improvement and had short waiting time in HAART. This might be related to the case that elders are non-adherent as compared to youngsters [24]. On the other hand, it is known that as patient's age increased, his/her CD4 cell count decreased and such a patient might be defaulted because of death and other reasons. Previous research indicates that CD4 cell count change had been affected by sex, clinical stages and educational levels [15]. Females had a better CD4 cell change improvement as compared to males; this might be the reason that females have good experience in prenatal and postnatal healthcare follow-ups as compared to males. Females also had experience in taking pills for family planning. This experience made female patients to be adherent to the prescribed medication properly and to have long waiting time in HAART program as compared to males. As the number

Table 8 Average fluctuation of CD4 cell count and Hazard Ratio estimates for final selected joint models

\begin{tabular}{|c|c|c|c|c|}
\hline \multirow[t]{2}{*}{ parameter } & \multicolumn{2}{|l|}{ Parameter estimate } & \multicolumn{2}{|c|}{ Hazard Ratio (HR) estimate. } \\
\hline & Average fluctuation & 95\% Credible interval & $\mathrm{HR}$ & 95\% Credible intervals \\
\hline Intercept & -4.350 & $(-7.435,-2.856)$ & 0.1295 & $(0.082,0.454)$ \\
\hline Age & -0.006 & $(-0.003,-0.019)$ & 0.0362 & $(0.009,0.052)$ \\
\hline Weight & 0.001 & $(-0.008,0.002)$ & 0.0056 & $(0.001,0.008)$ \\
\hline Baseline CD4 count & 0.004 & $(0.001,0.025)$ & 0.0015 & $(0.001,0.002)$ \\
\hline \multicolumn{5}{|c|}{ Marital Status (Reference = without partner) } \\
\hline With partner & 0.007 & $(0.003,0.009)$ & 0.005 & $(0.003,0.008)$ \\
\hline \multicolumn{5}{|l|}{ Sex (Reference = male) } \\
\hline Female & 0.034 & $(0.004,0.067)$ & 0.023 & $(0.003,0.075)$ \\
\hline \multicolumn{5}{|c|}{ Ownership of cell phone (Reference = With phone) } \\
\hline Without cell phone & -0.006 & $(-0.004,-0.024)$ & 0.007 & $(0.004,0.009)$ \\
\hline \multicolumn{5}{|c|}{ Level of adherence (Reference = Good adherence) } \\
\hline Poor adherence & -0.523 & $(-0.644,-0.235)$ & 0.483 & $(0.002,0.621)$ \\
\hline Fair adherence & -0.452 & $(-0.671,-0.253)$ & 0.552 & $(0.092,0.831)$ \\
\hline \multicolumn{5}{|c|}{ Level of disclosure of the disease (Reference = yes) } \\
\hline No & -0.002 & $(-0.073,-0.001)$ & 0.1020 & $(0.003,0.324)$ \\
\hline \multicolumn{5}{|c|}{ WHO stages (Reference $=$ Stage IV) } \\
\hline Stage I & 0.151 & $(-0.002,0.246)$ & 0.2511 & $(0.081,0.452)$ \\
\hline Stage ॥ & 0.157 & $(0.032,0.224)$ & 0.1567 & $(0.092,0.421)$ \\
\hline Stage III & 0.138 & $(-0.231,0.231)$ & 0.4381 & $(0.231,0.643)$ \\
\hline Time & 0.021 & $(0.001,0.064)$ & 0.1209 & $(0.091,0.241)$ \\
\hline$\tau_{1}$ & -2.965 & $(-4.547,-1.234)$ & 0.0341 & $(0.010,0.067)$ \\
\hline$T_{3}$ & 0.653 & $(0.254,0.923)$ & 1.7342 & $(1.234,1.966)$ \\
\hline
\end{tabular}


of follow-ups for patients increased, the defaulting rate of patients from the HAART program decreased. This might be happen since patients with long visiting time acquire more experience to adhere to the medication and this leads to improve their CD4 cell count progression and such patients might have long waiting time (greater number of follow-ups) in the HAART program. Patients with ownership of cell phone had a better CD4 cell count progress (growth) as compared to those without cell phone. Patients who used cell phone as memory aid had a better CD4 cell count progress/low CD4 cell count fluctuation. This memory aid helps to take pills on time and this has its own impact on the progress of the CD4 cell count and on the longevity of patients in the HAART program.

\section{Conclusion}

Certain groups that require intervention had been identified in the current research and these groups need special attention for the longevity of their life within HAART. An integrated intervention becomes effective for the patients to survive for long period of time with in the treatment. Ministry of Health or health staff should advise the patients to adhere to the prescribed medication properly to improve their CD4 cell count change/progress and to have a long time to default from HAART program. Health education should be given to patients who are living in rural areas, and who came after a declined number of CD4 cell count for diagnosis. Medical advice should also been given to patients to disclose the disease to get social support from families and communities and to have longer waiting time within the treatment.

The current research had limitations that the results obtained using a Bayesian approach may be different if likelihood approaches would be included and triangulated. This gap might be considered as potential area for future researchers. The data had been taken from one treatment site. If such data would be collected from different health institutions, the results may be different. The results of the current investigation are useful to make integrated intervention in providing health education to the patients, and would help to guide the policy and management of HAART. Further studies are recommended with additional predictors such as nutrition, religion, and consumption of substances on CD4 cell count change. The quality of health service provision which is not included under this study may have direct or indirect effects on patients' CD4 cell count change and this also needs further investigation in for future researches.

\section{Abbreviations}

CD4: Classification Determinant Four; DIC: Deviance information criteria; GLMM: Generalized Linear mixed model; HAART: Highly Active Ant-Retroviral Therapy; HIV: Human Immune Deficiency Virus; MCAR: Missed completely at random; OR: Odds Ratio; WHO: World Health Organization

\section{Acknowledgements}

The Amhara Region Health Research \& Laboratory Center at Felege-Hiwot Teaching and Specialized Hospital and all the health staffs are gratefully acknowledged for the data they supplied for current research.

\section{Availability of data and materials}

The data supporting these findings, found in the corresponding author, will not be made available publically due to concerns for protecting participants' identity and respecting their rights to privacy. At the time the study was conducted, the investigator did not obtain informed consent from participants for publication of the dataset.

\section{Authors' contributions}

AST wrote the proposal, developed data collection format, supervised the data collection process, analyzed and interpreted the data. PN and TZ participated in the design and data analysis, and critically read the manuscript and gave constructive comments for the betterment of the manuscript applying their rich experience. Finally, all authors approved the manuscript to be submitted to BMC infectious disease.

\section{Authors' information}

AST is an assistant professor of statistics department at Bahir Dar University, Ethiopia with four publications previously. Currently, he is a doctoral student in statistics department entitled "Statistical methods for analysis of longevity of medication on basis of monthly HAART adherence treatment response in North West Ethiopia (Amhara region)" with the close supervision of the second and third authors. PN is a senior associate professor of the University of South Africa with more than 10 publications. $\mathrm{TZ}$ is a senior and full professor of college of mathematics, Statistics and Computer science at university of Kwazulu Natal. He has more than sixty (70) publications in reputable journals.

All the three authors together had three publications using the same data with the current one and on the same study area. This will be the fourth article for our PhD paper. The previous three articles are;

1. First month CD4 cell count (Seyoum et al. AIDS Res Ther (2016) 13:36)

2. Joint modeling of CD4 cell count and Adherence to HAART (Seyoum et al. AIDS Res Ther (2017) 14:14).

3. Factors affecting first month adherence due to antiretroviral therapy among HIV positive adults at Felege Hiwot Teaching and Specialized Hospital, north-western Ethiopia; a prospective study (Tegegne et al., BMC infectious disease (2018) 18:83))

Ethics approval and consent to participate

The data was collected previously by the health staff for providing treatment/ to start HAART. The study was approved by the two committees namely Bahir Dar University's Ethical Approval committee, Bahir Dar University, Ethiopia with reference number, Ref $\neq$ RCS/1412/2006 and School of Science Research Ethics Review Committee (which belongs to University of South Africa, UNISA), South Africa with reference number, Ref : 2015 - SSR - ERC_006.

\section{Competing interests}

There is no financial and non-financial competing interest between authors and between authors and institutions.

\section{Publisher's Note}

Springer Nature remains neutral with regard to jurisdictional claims in published maps and institutional affiliations.

\section{Author details}

'Department of statistics, Bahir Dar University, Bahir Dar, Ethiopia. ${ }^{2}$ Department of Statistics, Unisa, Pretoria, South Africa. ${ }^{3}$ School of Mathematics, Statistics and Computer Science, University of Kwazulu Natal, Durban, South Africa. 
Received: 5 November 2017 Accepted: 20 April 2018

Published online: 27 April 2018

\section{References}

1. East S, Africa S-S. Towards universal access: scaling up priority HIV/AIDS interventions in the health sector. Europe. 2010;85:000.

2. Organization, W.H. Global Health risks-mortality and burden of disease attributable to selected major risks. Lancet. 2015;83:324.

3. Hladik W, et al. HIV/AIDS in Ethiopia: where is the epidemic heading? Sex Transm Infect. 2006;82(suppl 1):i32-5.

4. Berhan Z, et al. Prevalence of HIV and associated factors among infants born to HIV positive women in Amhara region, Ethiopia. Int J Clin Med. 2014;5(8):464.

5. Gezie LD. Predictors of CD4 count over time among HIV patients initiated ART in Felege Hiwot referral hospital, Northwest Ethiopia: multilevel analysis. BMC Res Notes. 2016;9(1):377

6. Organization, W.H., Antiretroviral therapy for HIV infection in adults and adolescents: recommendations for a public health approach-2010 revision. 2010.

7. Kaufmann GR, et al. CD4 T-lymphocyte recovery in individuals with advanced HIV-1 infection receiving potent antiretroviral therapy for 4 years: the Swiss HIV cohort study. Arch Intern Med. 2003;163(18):2187-95.

8. Florence $\mathrm{E}$, et al. Factors associated with a reduced CD4 lymphocyte count response to HAART despite full viral suppression in the EuroSIDA study. HIV Med. 2003;4(3):255-62.

9. Asfaw A, et al. cd4 cell count trends after commencement of antiretroviral therapy among HIV-infected patients in Tigray, northern Ethiopia: a retrospective cross-sectional study. PLoS One. 2015;10(3):e0122583.

10. Smith CJ, et al. Factors influencing increases in CD4 cell counts of HIVpositive persons receiving long-term highly active antiretroviral therapy. J Infect Dis. 2004;190(10):1860-8.

11. Gea-Banacloche JC, Clifford LH. Immune reconstitution in HIV infection. AIDS (London, England). 1998;13:S25-38.

12. Ebonyi $A O$, et al. Factors associated with a low CD4 count among HIV-1 infected patients at enrolment into HAART in Jos, Nigeria. Br J Med Med Res. 2014;4(13):2536.

13. Gandhi RT, et al. Effect of baseline-and treatment-related factors on immunologic recovery after initiation of antiretroviral therapy in HIV-1positive subjects: results from ACTG 384. J Acquir Immune Defic Syndr. 2006:42(4):426-34.

14. Seyoum A, Ndlovu P, Zewotir T. Quasi-Poisson versus negative binomial regression models in identifying factors affecting initial CD4 cell count change due to antiretroviral therapy administered to HIV-positive adults in north-West Ethiopia (Amhara region). AIDS Res Ther. 2016;13(1):36.

15. Seid A, et al. Joint modeling of longitudinal CD4 cell counts and time-todefault from HAART treatment: a comparison of separate and joint models. Electron J Appl Stat Anal. 2014;7(2):292-314.

16. Faes C, Geys H, Catalano P. Joint models for continuous and discrete longitudinal data. Longitudinal Data Anal. 2009;327:348.

17. Muenz LR, Rubinstein LV. Markov models for covariate dependence of binary sequences. Biometrics. 1985;13:91-101.

18. Guo X, Carlin BP. Separate and joint modeling of longitudinal and event time data using standard computer packages. Am Stat. 2004;58(1):16-24

19. DiMatteo MR. Variations in patients' adherence to medical recommendations: a quantitative review of 50 years of research. Med Care. 2004;42(3):200-9.

20. Hedeker D, Gibbons RD. Application of random-effects pattern-mixture models for missing data in longitudinal studies. Psychol Methods. 1997;2(1):64.

21. Keselman $\mathrm{H}$, et al. A comparison of two approaches for selecting covariance structures in the analysis of repeated measurements. Commun Stat Simul Comput. 1998;27(3):591-604.

22. Pan W. Akaike's information criterion in generalized estimating equations. Biometrics. 2001;57(1):120-5.

23. Gardner W, Mulvey EP, Shaw EC. Regression analyses of counts and rates: Poisson, overdispersed Poisson, and negative binomial models. Psychol Bull. 1995:118(3):392.

24. Viard J-P, et al. Influence of age on CD4 cell recovery in human immunodeficiency virus-infected patients receiving highly active antiretroviral therapy: evidence from the EuroSIDA study. J Infect Dis. 2001; 183(8):1290-4

\section{Ready to submit your research? Choose BMC and benefit from:}

- fast, convenient online submission

- thorough peer review by experienced researchers in your field

- rapid publication on acceptance

- support for research data, including large and complex data types

- gold Open Access which fosters wider collaboration and increased citations

- maximum visibility for your research: over $100 \mathrm{M}$ website views per year

At BMC, research is always in progress.

Learn more biomedcentral.com/submissions 